\title{
Adapting the Clinical MRI Software Environment for Real-Time Navigation of an Endovascular Untethered Ferromagnetic Bead for Future Endovascular Interventions
}

\author{
Arnaud Chanu, ${ }^{1}$ Ouajdi Felfoul, ${ }^{1}$ Gilles Beaudoin, ${ }^{2}$ and Sylvain Martel ${ }^{1 *}$
}

\begin{abstract}
A dedicated software architecture for a novel interventional method allowing the navigation of ferromagnetic endovascular devices using a standard real-time clinical MRI system is shown. Through a specially developed software environment integrating a tracking method and a real-time controller algorithm, a clinical 1.5T Siemens Avanto MRI system is adapted to provide new functionality for potential automated interventional applications. The proposed software architecture was successfully validated through in vivo controlled navigation inside the carotid artery of a swine. Here we present how this MRI-upgraded software environment could also be used in more complex vasculature models through the real-time navigation of a $1.5 \mathrm{~mm}$ diameter chrome steel bead in two different MR-compatible phantoms with flowless and quiescent flow conditions. The developed platform and software modules needed for such navigation are also presented. Real-time tracking achieved through a dedicated positioning method based on an off-resonance excitation technique has also been successfully integrated in the software platform while maintaining adequate real-time performance. These preliminary feasibility experiments suggest that navigation of such devices can be achieved using a similar software architecture on other conventional clinical MRI systems at an operational closed-loop control frequency of $32 \mathrm{~Hz}$. Magn Reson Med 59:1287-1297, 2008. (c) 2008 Wiley-Liss, Inc.
\end{abstract}

Key words: real-time navigation; ferromagnetic device; software architecture; magnetic propulsion

Because MR imaging offers many advantages in term of spatial resolution and safety for the patient, modern interventional medicine is now considering this imaging modality as an alternative for upcoming interventional applications. Many standard fluoroscopy catheter procedures can now be executed in an MRI environment (1-3), providing enhanced visual precision for the medical team without patient exposure limitations due to radiation emission. However, all catheter-based procedures,

${ }^{1}$ NanoRobotics Laboratory, Department of Computer Engineering and Institute of Biomedical Engineering, École Polytechnique de Montréal (EPM), Campus of the University of Montréal, Montréal, Canada.

2University of Montréal, Montréal, Canada.

Grant sponsor: Canada Research Chair (CRC) in Micro/Nanosystem Development, Fabrication and Validation; Grant sponsor: Canada Foundation for Innovation (CFI); Grant sponsor: National Sciences and Engineering Research Council of Canada (NSERC); Grant sponsor: Government of Québec.

${ }^{*}$ Correspondence to: S. Martel, NanoRobotics Laboratory, Department of Computer Engineering and Institute of Biomedical Engineering, École Polytechnique de Montréal (EPM), Campus of the University of Montréal, Montréal, QC H3C 3J7, Canada. E-mail: sylvain.martel@polymtl.ca)

Received 20 December 2006; revised 19 January 2008; accepted 20 February 2008.

DOI 10.1002/mrm.21638

Published online in Wiley InterScience (www.interscience.wiley.com).

(c) 2008 Wiley-Liss, Inc. whether offering or not a better quality in visualization, face the same limitations with regard to accessibility and flexibility. Many actual medical interventions would require enhanced targeting of specific organs or physiological sites for the delivery of drug or contrast agents (4-7). Although catheters can reach some of these specific sites without much complication, many sites remain inaccessible and cannot be reached without involving major invasive surgeries. Moreover, these interventional applications rely on MRI capabilities only for the imaging modality provided by the system without considering using an MRI system as a fully integrated and automated endovascular navigation environment.

A recent breakthrough in interventional MRI-guided in vivo procedures demonstrated that real-time MRI systems can offer a well-suited integrated environment for the propulsion, tracking, and control of a ferromagnetic device, which was done in the carotid artery of a living swine (8). The standard imaging environment, however, must be adapted in order to provide such new capabilities. Timing and integration constraints must be overcome to permit the use of a standard MRI system as a new endovascular navigation platform. Unlike some other methods that simply use a DC field flux to create a torque on medical tools $(9,10)$, the present platform exploits the magnetic gradient provided by the MRI gradient coils to create a mechanical force that can be oriented in any desired direction to drive a magnetic body or particle to a given destination in the cardiovascular system (11-13). With such a driving mechanism tightly integrated with a dedicated ferromagnetic tracking technique and a navigation controller, magnetic bodies or particles could be driven for directional controlled target drug delivery or for navigable magnetic carriers for implantable biosensors.

In this study we present the requirements necessary to provide this new functionality for real-time clinical MRI systems. Through experimental navigation tests on two different $\mathrm{MR}$ phantoms with a $1.5 \mathrm{~mm}$ diameter chrome steel bead we show that most clinical MRI systems can be adapted to perform such new functionality.

\section{MATERIALS AND METHODS}

Description of the Platform

The real-time navigation of a ferromagnetic core in the human blood vessels implies the capability to propel, track, and control the device at an appropriate sampling frequency in a given direction contained in a complex 
fluidic environment. The propulsion method is based on Ref. (14) and uses the magnetic gradient coils of the MRI system to apply a magnetic force with a given magnitude and direction. From Ref. (14) we show that the magnetic force acting on the core is:

$$
\mathbf{F}_{\mathbf{m}}=\mathbf{V}_{\mathrm{m}} \cdot(\mathbf{M} \cdot \nabla) \mathbf{G} \cdot \boldsymbol{R} .
$$

where $V_{m}$ is the volume of the ferromagnetic core, $\mathbf{M}$ its magnetization, $\nabla$ the gradient operator, $\mathbf{G}$ the magnetic gradient amplitude, and $R$ the duty cycle during which the magnetic gradient is applied. In order for the device to be guided to reach a given location, a dedicated platform that integrates software modules for tracking, control, propulsion, and path planning within technological, physiological, and specific time constraints is essential. Such modules must be able to communicate seamlessly with each other at specific time in order to be able to react to the environment's evolution such as, but not limited to, blood flow variation or potential path trajectory correction due to various perturbations including but not limited to patient movement or breathing.

For instance, the 1.5T Siemens Avanto system (Malvern, PA) used for the experimental results provides three distinct computers responsible for the sequence execution. The first computer is the 'Measurement and Physiological Control Unit' (MPCU) and is responsible for the sequence events to be prepared before being sent to the scanner. Such events are common MR pulse sequence elements such as gradients, RF pulses, and apparent diffusion coefficient (ADC) for signal measurements. The second computer is the 'MR Image Reconstruction' (MRIR) and is responsible for the image calculation process during or after the sequence execution through the execution of a reconstruction routine. The third computer is the scanner itself on which are played the pulse sequence events. Most clinical MRI systems are now designed with a real-time feedback capability that allows the image reconstruction process to react on the running sequence by changing certain pulse events parameters at runtime. This new technology is well exploited in interventional MRI applications $(15,16)$ where medical procedures usually require scanning plane orientation to be changed on the fly in order to maintain medical tools in sight. The method described here implies using this real-time feedback capability to continuously change the magnetic gradient orientation and amplitude in order to drive the magnetic core to a given position. A feedback loop between the image reconstruction process and the running sequence is thus created, allowing sequence runtime modification to take place depending on the result of the acquired data coming from the sequence measurements. Since the reconstruction routine is responsible for the sequence upcoming events in the case that a real-time feedback loop is present, all the developed decision algorithms and modules are implemented to run on the image reconstruction computer.

Four main modules have been established for the device's navigation. A tracking module is responsible for data analysis and device position calculation based on the acquired data coming from the tracking phase within the real-time sequence. A propulsion and controller module takes care of the command calculation to determine a magnetic gradient amplitude and direction to be applied in the next propulsion phase of the running pulse sequence. The third module is the path planning responsible for the user-defined waypoints generation used in the controller module for the command computation. All these software modules are operated through a main central module known as the agent.

The agent is responsible for real-time data communication between the reconstruction routine and the running sequence. The dedicated software architecture lies in a standard pulse sequence image reconstruction routine. Figure 1 shows the overall software platform architecture with the pulse sequence environment on the right and the image reconstruction routine on the left. The real-time feedback loop is illustrated in the center of Fig. 1, linking the image reconstruction routine to the running sequence. This software architecture is the necessary bonding element that allows a seamless integration of the different aspects of the navigation process.

\section{Dynamic Tracking}

Ferromagnetic materials cause significant magnetic susceptibility artifacts (17-19) when present inside the MRI during imaging. Although special imaging techniques for magnetic susceptibility artifact correction such as view angle tilting (20) exist, image acquisition delays offer a great challenge for real-time interventional MRI. The tracking method used herein is based on an off-resonance excitation technique (21) that allows 3D tracking of a ferromagnetic device in $\approx 22 \mathrm{~ms}$ using three orthogonal projections. Referred to as magnetic signature selective excitation tracking (MS-SET), this method consists of exciting protons and using the induced magnetic equipotential curves generated by the ferromagnetic core through an off-resonance excitation for the acquisition of three orthogonal $k$-space lines. The position is obtained by correlating a given projection at a given time with the ones previously obtained. The absolute position could be acquired only at the beginning of the experiment, where it is used as a reference for subsequent relative positions or during the intervention in order to refresh the absolute position $(22,23)$.

The sequence used for this study is based on a gradient echo implementation modified from the one presented in Ref. (23). In fact, gradient echo MS-SET allows a higher positioning refresh rate than spin echo. The three orthogonal projections are executed with a single RF pulse tuned to excite protons surrounding the bead or the device being controlled. Figure 2 illustrates the tracking sequence overview. Small gradients orthogonal to the readout direction are used in order to compensate the rapid signal decay caused by inhomogeneities of the magnetic field induced by the marker. To avoid gradient imbalance in the readout direction, the dephaser gradient played before the readout always accounts for the blips and cancel them. Note that this sequence does not contain a slice select nor phase direction, since the excitation is done through all the volume without the application of an external gradient given that we only acquire the central $k$-space line. A positive 


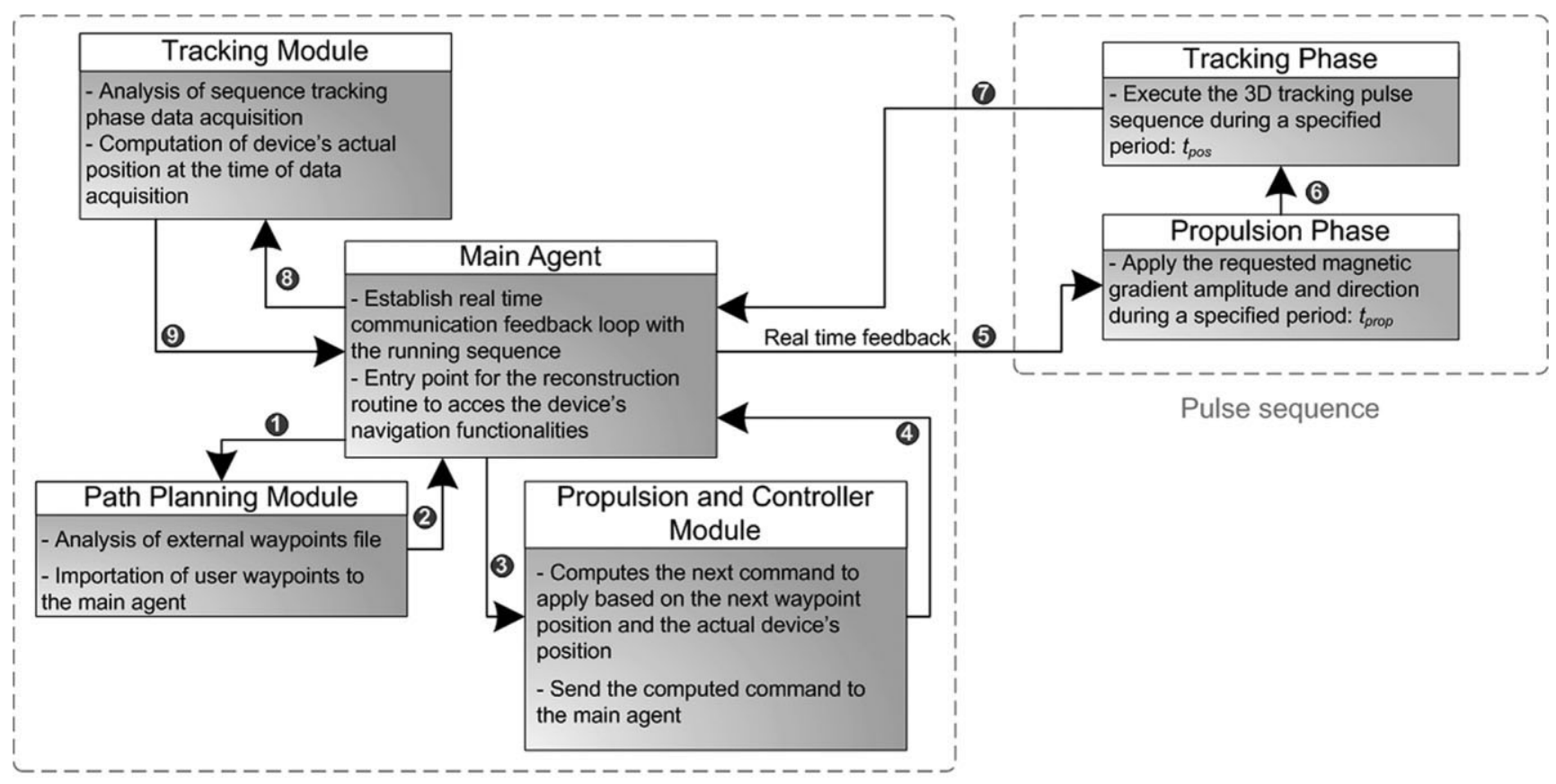

Image reconstruction routine

FIG. 1. Overview of the platform architecture. 1: Waypoint list request (done once). 2: Waypoint list sent to the main agent (done once). 3 : Device's position sent to the propulsion and controller module. 4: Controller's command computation sent to the main agent. 5: Command is sent to the running sequence for execution through real-time feedback. 6: Execution of the propulsion phase followed by the tracking phase. 7: Tracking data sent to the image reconstruction routine (main agent). 8: Device position computation based on last received measurement. 9: Device position sent to the main agent.

contrast is obtained by the same principles as the white marker phenomenon described in Ref. (24) but adapted for the case of $1 \mathrm{D}$ acquisition. In order to minimize the effect of the gradient fields used to measure the position along the displacement of the ferromagnetic object, gradient moments are null along each axis, except for the dephaser and spoiler at the end of the sequence kernel.

The accuracy of determining the position of the bead is found to be approximately the size of a pixel for a moving sphere and half a pixel for a static sphere (21). The signalto-noise ratio (SNR) of the tracking sequence depends on the sequence parameters, and the properties of the bead and the background. In our experiments we used a flip angle ranging from $15^{\circ}$ to $30^{\circ}$, as well as the standard RF body array coils provided with the MRI system. RF frequencies offset ranging from 1-2 kHz were used in order to excite the proton spins. An acceptable SNR between 15 and 20 was obtained from the projections data along the $x$ and $y$-axis and $\approx 10$ for the $z$-axis, as illustrated in Fig. 3 . Bead detection on the z-axis is ambiguous because of the multiple peaks in the correlation function. However, the peak corresponding to a match between the two correlated projections was higher than the two others and special care was taken in order not to choose local maxima in the correlation function. The tracking accuracy was $1.17 \mathrm{~mm}$, with every tracking projection acquiring 256 points within a 300-mm field of view (FOV).

\section{Real-time Sequence}

The pulse sequence designed for the ferromagnetic device navigation is illustrated in Fig. 4. Three main events com- pose a standard acquisition denoted 'Repetition' in Fig. 4. Such acquisition is repeated until the device has reached its final target destination. The synchronization event, denoted 'Sync Event' in Fig. 4, is the first event of the sequence and is responsible for triggering the execution of the real-time controller located in the image calculation routine of the MRIR. This routine computes the new command to be applied in the next repetition and sends it back to the running sequence through the main agent, as illustrated by the arrow denoted 'Control Command' in Fig. 4. In order for the real-time controller routine to complete, a real-time feedback delay must be allowed to prevent navigation abortion. During this real-time feedback delay the pulse sequence waits for the new gradient command to be received and is considered idle since no pulse event is executed. In order to have the shortest repetition time, the present implementation of the pulse sequence has been designed so that this idle delay is filled up by propulsion and tracking events. The second event is the propulsion block which applies the last computed command on the scanner. This command is a set of three gradient magnitudes, one for each direction. However, the available magnetic force created for the propulsion of the device is limited in time and amplitude because of the heat dissipation threshold of the magnetic coils. It is possible to reduce such heat generation and, therefore, gradient application time by introducing a pulsating gradient. In this case the duty cycle determines the on and off times of the gradient, as noted in Eq. [1]. The third event block is the tracking sequence and will be explained in detail below. During its execution the three projection data are acquired 


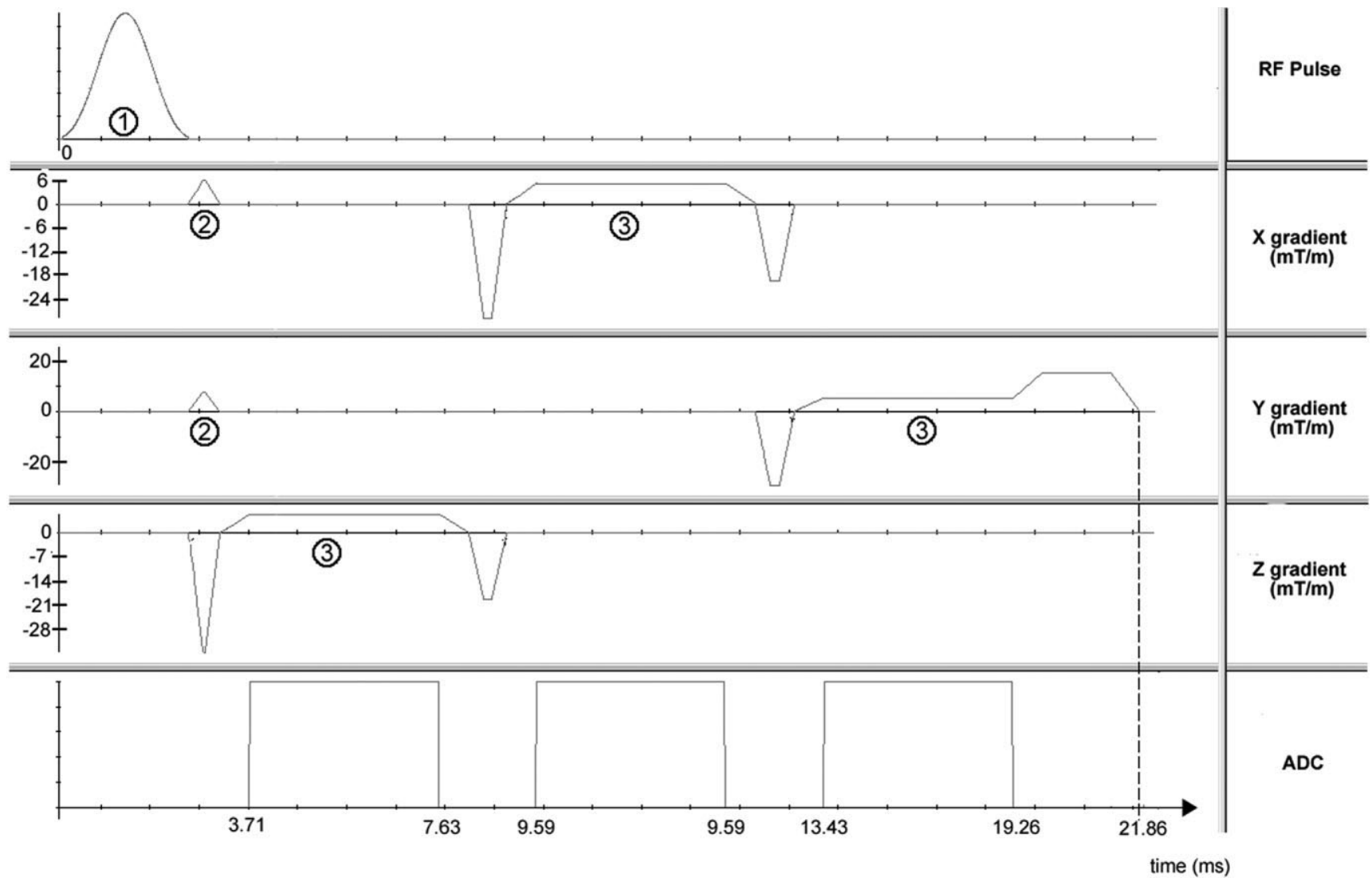

FIG. 2. Overview of the tracking pulse sequence. 1) A nonselective RF pulse tuned to the desired frequencies regarding the induced magnetic field from the ferromagnetic object is applied. 2) Short dephaser gradients are applied in the direction orthogonal to the read one in order to compensate the rapid signal decay caused by inhomogeneities of the magnetic field induced by the marker. A single RF pulse serves for the acquisition of three projections allowing for the tracking of the device in less than 30 ms. 3) Readout gradients are successively applied on each axis $z, x$, and $y$ in order to obtain the projections data.

and sent to another running process in the image calculation routine which takes care of data analysis and correlation computation leading to the three final device displacement values on each axis.

The real-time feedback delay is chosen by the operator before the sequence is initiated. The minimum feedback delay must be obtained empirically as it depends, among other things, on the complexity of the real-time control routine executed in the image calculation program. From Fig. 4 we see that the real-time feedback delay is simply defined as:

$$
\mathbf{t}_{\text {feedback }}=\mathbf{t}_{\text {propulsion }}+\mathbf{t}_{\text {tracking }}
$$

where $t_{\text {tracking }}$ is the tracking sequence duration (22 ms) and $t_{\text {propulsion }}$ is the propulsion block duration.

\section{Control}

The real-time controller designed for the error computation and the command generation is based on a simple 2D proportional, integral, derivative (PID) algorithm (25). The
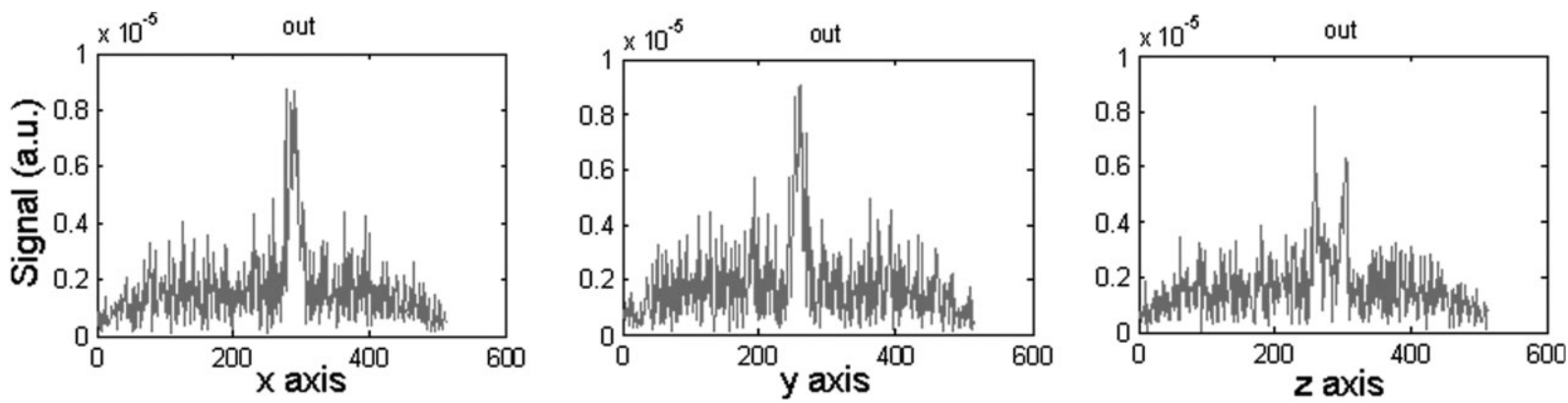

FIG. 3. Projections data for the $x, y$, and $z$ axis. The computed SNR depends on the background tissue but has a mean value ranging from 15 to 20 for the $x$ and $y$ axis and slightly above 10 for the $z$ axis. 


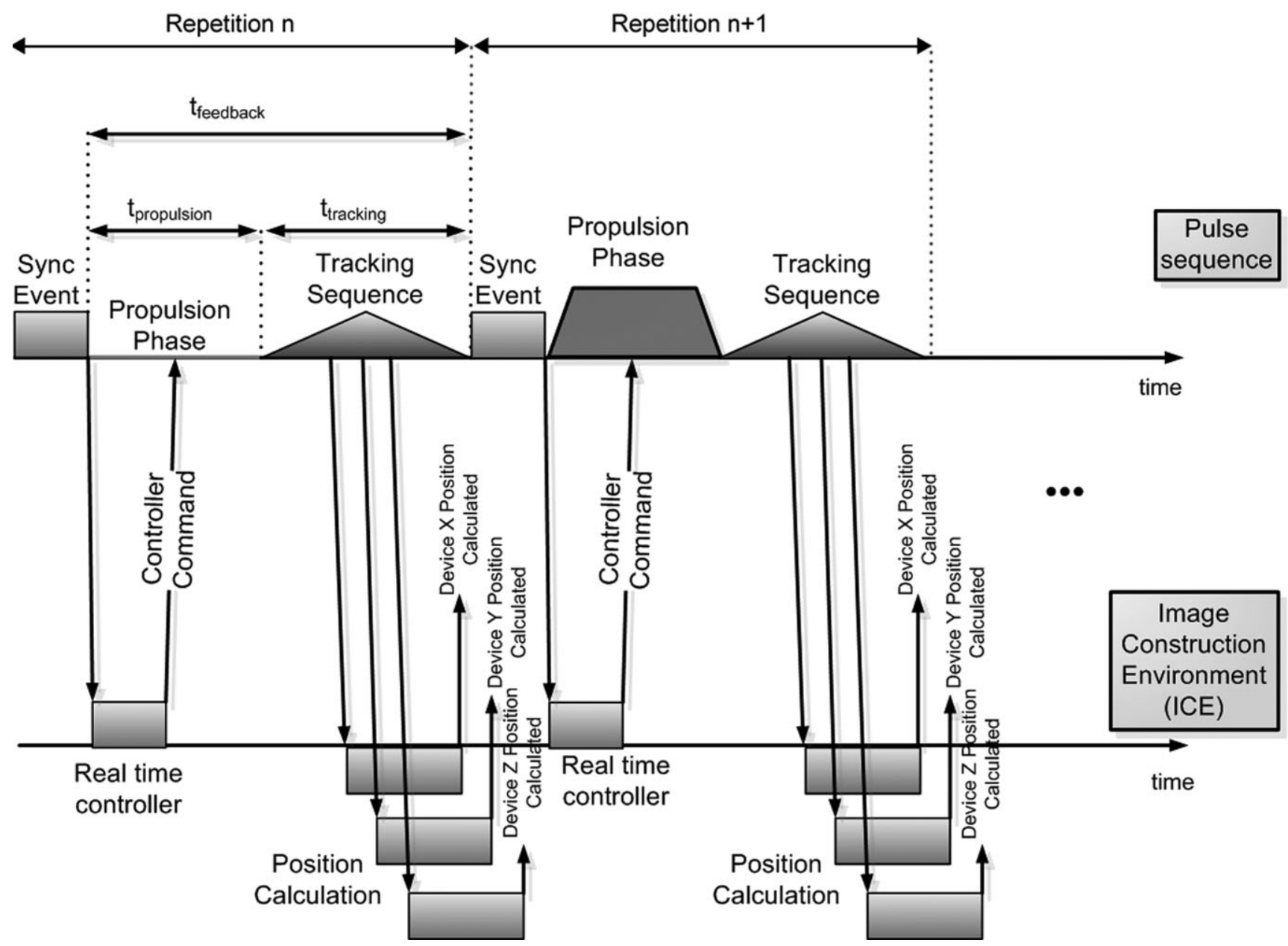

FIG. 4. Overview of the complete real-time pulse sequence used for the device's navigation.

maximum magnetic gradient amplitude allowed by the $1.5 \mathrm{~T}$ Siemens Avanto ( $80 \mathrm{mT} / \mathrm{m}$ peak to peak) is not sufficient for the levitation of the $1.5 \mathrm{~mm}$ diameter chrome steel bead used in our experiments, whether it is placed in water or blood (14). According to Ref. (14), the required force to levitate a ferromagnetic device in a vertical blood vessel in the absence of flow is described as:

$$
\mathbf{F}_{\text {MagLev }}=-(\mathbf{W}-\mathbf{b})
$$

where $\mathrm{W}$ is the weight of the device and $\mathrm{b}$ the buoyancy. A constant mean magnetic gradient amplitude of $61 \mathrm{mT} / \mathrm{m}$ would be necessary in order to levitate the bead inside an environment made of water.

For this reason, only a 2D controller is considered in this article. But as the size of the ferromagnetic device decreases, such as for navigation in smaller diameter blood vessels, gravitational force becomes less significant compared to other forces and, as such, a 3D controller can be considered. But for a larger ferromagnetic entity, the model considered here for the 2D navigation of the $1.5 \mathrm{~mm}$ bead in an in vitro fluidic environment is described in Refs. (25-27) and consists of five major forces acting on the bead: the magnetic force generated by the gradient coils as expressed in Eq. [1], the fluid drag force, the weight of the bead, the buoyancy force, and the sliding friction force. A simple 2D model representation is illustrated in Fig. 5. Recent in vivo navigation of a $1.5 \mathrm{~mm}$ diameter chrome steel bead in the carotid artery of a living swine was conducted in order to validate the model (8) and a quantitative force evaluation is provided below.

Since the propulsion of microdevices in terms of control algorithms and propulsion efficiency highly depends on

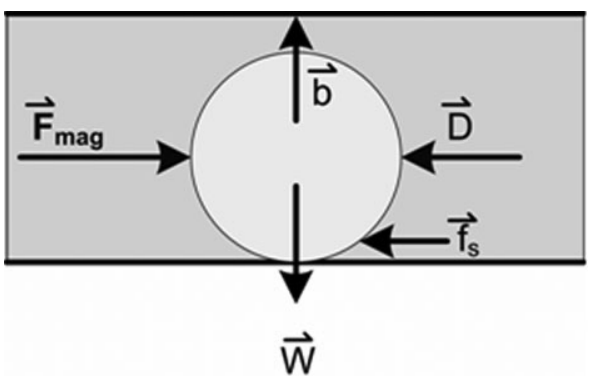

FIG. 5. Illustration of the simple force model for the navigation of the device in a blood vessel. For simplicity considerations, only the $2 \mathrm{D}$ model is shown. $F_{\text {mag }}$ is the magnetic force produced by the magnetic gradient, $b$ the buoyancy force, $W$ the weight, $D$ the drag force, and $\mathrm{f}_{\mathrm{s}}$ is the sliding or rolling friction force depending on the orientation of the displacement. 
the diameter of the blood vessels (14) and to avoid any need for hardware modifications or upgrades on these standard MRI platforms, only macro-scale controlled navigation such as in arteries or veins is considered initially.

In the software platform the controller algorithm responsible for such navigation is located in the image calculation environment, inside the reconstruction routine. As illustrated in Fig. 4, the real-time controller is called at the end of the synchronization event in the pulse sequence through the main agent. During its execution the controller routine reads the last computed device position acquisition from the tracking module and computes the $\mathrm{x}, \mathrm{y}$, and $\mathrm{z}$ errors between the last position of the device and the targeted waypoint. The overall distance between the actual position and the waypoint is computed as:

$\Delta_{x, y, z}=$
$\quad \sqrt{\left(x_{\text {waypoint }}-x_{\text {device }}\right)^{2}+\left(y_{\text {waypoint }}-y_{\text {device }}\right)^{2}+\left(z_{\text {waypoint }}-z_{\text {device }}\right)^{2}}$.

where $x_{\text {waypoint }}, y_{\text {waypoint }}$, and $z_{\text {waypoint }}$ are respectively the $\mathrm{x}$, $y$, and $z$ coordinates of the next targeted waypoint to be reached and $x_{\text {device }}, y_{\text {device }}$, and $z_{\text {device }}$ are respectively the last acquired $\mathrm{x}, \mathrm{y}$, and $\mathrm{z}$ coordinates of the device.

To determine if a given waypoint has been reached by the device, the architecture defines a virtual precision circle with a predefined radius $\phi_{p}$ around each waypoint. If at any given time the device's computed position is found to be inside the targeted waypoint precision circle, the controller module considers the waypoint as reached and loads the next waypoint as the new target. Once this is done, a new command consisting of $\mathrm{x}, \mathrm{y}$, and $\mathrm{z}$ gradients amplitude components is computed

\section{Experimental Setups}

The performance of the software architecture has been assessed by conducting navigation experiments in two distinct MR-compatible phantoms. The first phantom consists of a thin polymethylmethacrylate (PMMA) plate on which the device is free to move in the $\mathrm{x}$ and $\mathrm{z}$ plane with respect to the standard MRI Cartesian coordinate system. The thin PMMA plate is mounted on three threaded plastic rods screwed to a thick PMMA plate. Horizontality of the thin plate can be adjusted through the three nuts on each rod. A PMMA lid placed above the thin plate allows the ferromagnetic device to be contained within a $3 \mathrm{~mm}$ wide gap so the device can freely move on the plate. The hand-crafted phantom is placed in a water-filled container in order to obtain a homogeneous environment for proton excitation during the tracking phase of the pulses sequence. Figure 6 illustrates this first 2D MR setup.

The second MR phantom used for the 2D navigation experiments under quiescent flow consists of an MR human carotid replica (Shelley Medical Imaging Technologies, Toronto, Canada). As shown in Fig. 7, tubing on the sides of the replica allows connection to a mechanical pump (March Pumps, Glenview, IL) and a valve controlled flow meter device (Barnant Co., Barrington, IL). The mechanical pump and the valve are placed in the control room, connected to the carotid phantom in the scanner room with tubing passing through a small RF shielded opening. The flow rate ranged from 6 to $11 \mathrm{~cm} / \mathrm{s}$ with a mean value of $9 \mathrm{~cm} / \mathrm{s}$. Like the above-mentioned 2D plate phantom, the carotid replica was placed in a water-filled container, completely submerging it.

For all the navigation experiments the device considered is a ferromagnetic chrome steel bead of $1.5 \mathrm{~mm}$ diameter (Salem Specialty Ball, Canton, CT) with a mass density $\rho=8.41 \mathrm{~kg} / \mathrm{m}^{3}$. Using a Gaussmeter (Walker Scientific, Worcester, MA, MG-50, 10G to $10 \mathrm{kG}$ ) under an applied field $B_{0}=1.5 \mathrm{~T}$, the measured saturation magnetization of the bead was $M=1.35 \times 10^{6} \mathrm{~A} / \mathrm{m}$. The MS-SET tracking technique was used for the device positioning.

The waypoints positioning for the path planning operation was achieved using the external volume visualization application Volview 2.0 from Kitware (Clifton Park, NY).

\section{RESULTS}

\section{Real-time Navigation}

The current implementation of the controller routine allows a minimum feedback delay of $t_{\text {feedback }}^{\min }=30 \mathrm{~ms}$ below which the routine cannot complete in time, leading to a sequence stop. Considering such a minimum feedback delay and according to [2], the propulsion block duration is $t_{\text {propulsion }}^{\min }=8 \mathrm{~ms}$. The maximum feedback delay, on the other hand, is restricted by the gradient coil hardware heating internal limit, which in turn depends on the selected propulsion gradient duty cycle and the maximum gradient amplitude used during navigation of the device. During the experiments the maximum gradient amplitude on each axis was set to $40 \mathrm{mT} / \mathrm{m}$, allowing a maximum feedback delay of $39 \mathrm{~ms}$ with a $94 \%$ duty cycle for the propulsion gradients (considering the gradient rise time). This delay allows a propulsion time of $t_{\text {propulsion }}^{\max }=17 \mathrm{~ms}$. If a higher feedback delay is considered, the propulsion duty cycle must be lowered in order to accommodate a normal navigation sequence operation. Propulsion time is a major component for efficient navigation of the device. However, even though a longer real-time feedback delay implies a longer propulsion time, it also implies a lower overall operation frequency, which is also critical for the controller's stability and efficiency.

\section{Control}

Navigation and Control Parameters Common to All Experiments

The real-time feedback delay considered for the experiments was the minimum time allowed of $t_{\text {feedback }}^{\min }=30 \mathrm{~ms}$. The overall pulse sequence duration was thus $t_{\text {repetition }}=$

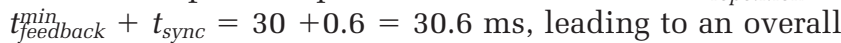
operational frequency of $32 \mathrm{~Hz}$. The 2D PID controller described in Ref. (27) is used for all the closed-loop control experiments presented in this section. This PID consists of a time-based error computation part which determines the quadratic error in $2 \mathrm{D}$ between the bead's current position and its destination as expressed in Eq. [4]. The error consists of a tangential and normal error component with respect to the ideal displacement vector between the pre- 


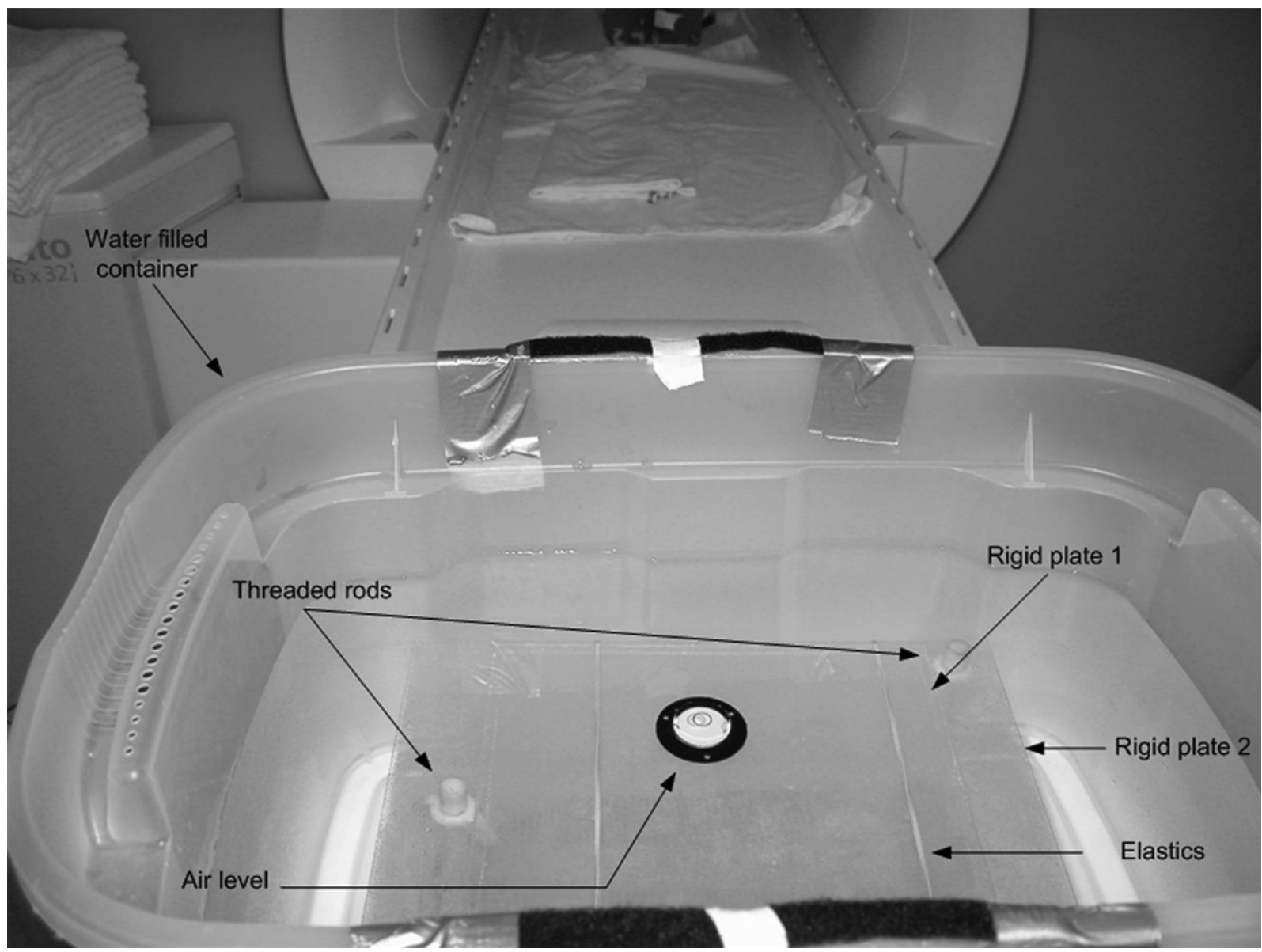

FIG. 6. 2D setup for flowless experiments. A PMMA rigid plate (Rigid plate 2 ) is screwed onto three threaded plastic rods (Threaded rods) and holds the ferromagnetic bead. A PMMA cover lid (Rigid plate 1) is placed above Rigid plate 1 in order to allow a $3 \mathrm{~mm}$ gap between both plates in which the bead is free to move in the $\mathrm{x}, \mathrm{z}$ plane. Horizontality is adjusted through the nuts on the rods and an air level placed onto the top lid. The setup is inserted in a water filled container and placed on the MRI table.

vious waypoint and the next waypoint. The command computed consists of a combined $\mathrm{x}$ and $\mathrm{z}$ magnetic gradient amplitudes to be applied on the bead to reach the next waypoint and to minimize the quadratic error.

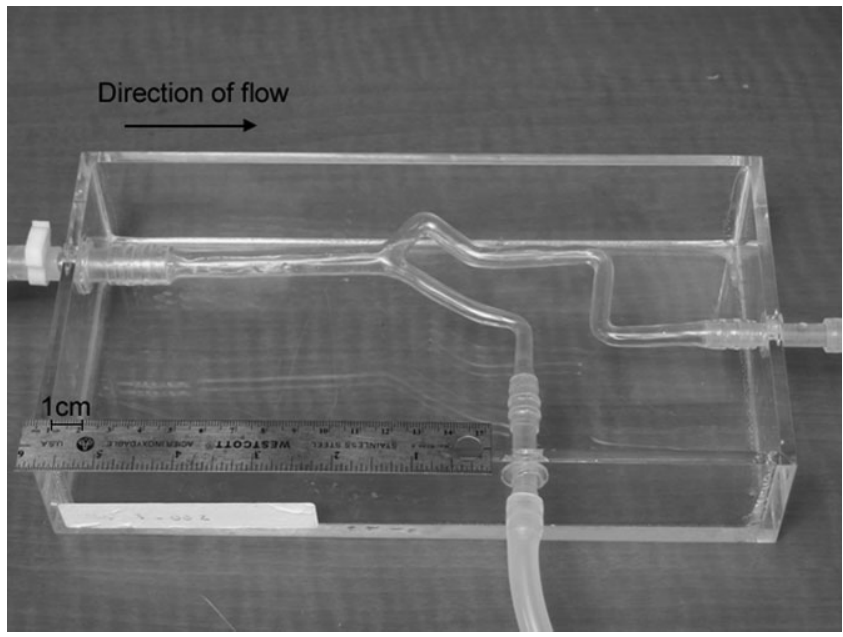

FIG. 7. Shelley human carotid phantom for quiescent flow experiments.
The bead's selected diameter was chosen in order to be optimal in terms of magnetic propulsion power and the diameter of the macro-vasculature used for navigation.

\section{$2 D$ Control on the MR Plate Phantom}

Before starting the navigation process, four waypoint positions were manually entered in the appropriate path file. Table 1 shows the different waypoint positions considered for the experiment. The user-defined precision sphere radius was set to $\phi_{p}=2 \mathrm{~mm}$. Figure 8 depicts the closedloop navigation experiments along the selected path. The number of repetitions for the experiment was set to 600 .

Table 1

Waypoint Positions for the 2D PMMA Plate Phantom

\begin{tabular}{cccc}
\hline $\begin{array}{c}\text { Waypoint } \\
\text { Numbering }\end{array}$ & $\begin{array}{c}\text { x Position } \\
(\mathrm{cm})\end{array}$ & $\begin{array}{c}\text { y Position } \\
(\mathrm{cm})\end{array}$ & $\begin{array}{c}\text { z Position } \\
(\mathrm{cm})\end{array}$ \\
\hline 1 & 2.0 & 0 & 2.0 \\
2 & 4.0 & 0 & 4.0 \\
3 & 6.0 & 0 & 6.0 \\
4 & 5.0 & 0 & 8.8
\end{tabular}




\section{$2 D$ Control in MR Carotid Phantom with Quiescent Flow}

Before the bead is injected, nine waypoints are placed through the external visualization application on a preacquired angiogram of the phantom. Table 2 presents the waypoint positions. The user-defined precision sphere radius was set to $\phi_{p}=10 \mathrm{~mm}$. This value, which is larger than the phantom's diameter size, was chosen in order to obtain a continuous valid region of precision even if the phantom slightly translates or vibrates following the application of gradients with high amplitude. The valve is adjusted to output a small quiescent flow directed from the main trunk of the phantom toward the central bifurcation. The number of repetitions is set to 2000. Figure 9 presents the selected path, with the chosen precision sphere radius illustrated by the circles. Figure 10 depicts in detail the experimental $\mathrm{x}$ and $\mathrm{z}$ data positions against time.

\section{DISCUSSION}

Magnetic Gradient Strength Limitation and Overcoming Possibilities

Using the available $40 \mathrm{mT} / \mathrm{m}$ magnetic gradient amplitude and considering a device magnetization and size as described above, the peak magnetic force acting on the device when propelled in the $\mathrm{x}$ or $\mathrm{z}$ direction is $\mathrm{F}_{\mathrm{mag}}=95.4$ $\mu \mathrm{N}$. The drag force acting on the device as described in Ref. (27) and illustrated in Fig. 5 is calculated considering the bead being immersed in water and sustaining a constant flow of $9 \mathrm{~cm} / \mathrm{s}$. The computed value is $\mathrm{D}=7.54 \mu \mathrm{N}$. The weight of the bead is $\mathrm{W}=145 \mu \mathrm{N}$, the buoyancy is $\mathrm{b}=$ $17.3 \mu \mathrm{N}$, and the sliding friction force is $\mathrm{f}_{\mathrm{s}}=0.00739 \mu \mathrm{N}$.

The preliminary platform described above implies the absence of hardware modification of any kind. Such constraint limits the available magnetic force to be applied on the magnetic device since only the image encoding magnetic gradient coils are used in order to drive the device. For this reason, the generated force on the 1.5T Avanto does not allow levitation of the $1.5 \mathrm{~mm}$ diameter ferromag-

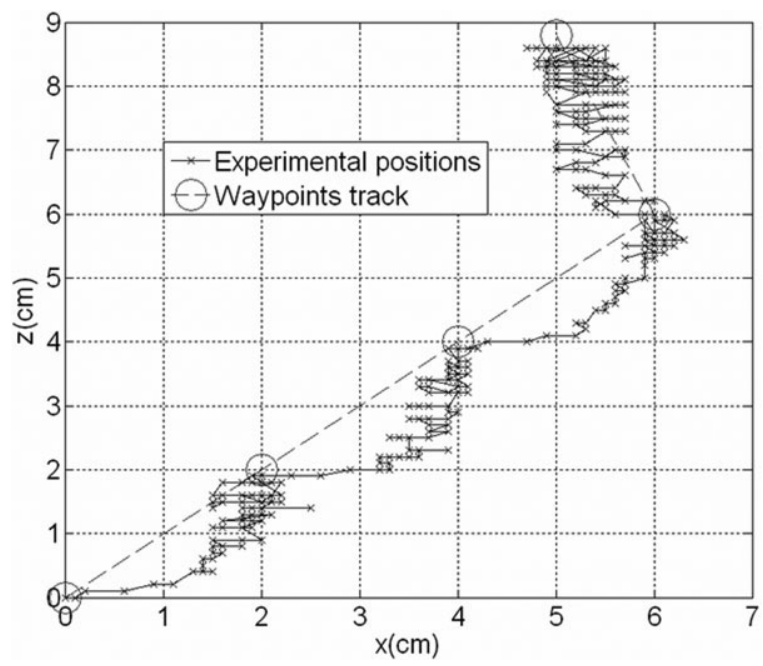

FIG. 8. Navigation experimental closed loop data along a path of waypoints on a PMMA plate.
Table 2

Waypoint Positions for the 2D Carotid Shelley Phantom

\begin{tabular}{cccc}
\hline $\begin{array}{c}\text { Waypoint } \\
\text { Numbering }\end{array}$ & $\begin{array}{c}\text { x Position } \\
(\mathrm{cm})\end{array}$ & $\begin{array}{c}\text { y Position } \\
(\mathrm{cm})\end{array}$ & $\begin{array}{c}\mathrm{z} \text { Position } \\
(\mathrm{cm})\end{array}$ \\
\hline 1 & 0 & 0 & 0.186 \\
2 & 1.74 & 0 & 0.186 \\
3 & 3.74 & 0 & 0.186 \\
4 & 5.74 & 0 & 1.11 \\
5 & 7.70 & 0 & 2.14 \\
6 & 4.48 & 0 & 0 \\
7 & 5.45 & 0 & -1.4 \\
8 & 3.74 & 0 & 0.186 \\
9 & 0 & 0 & 0.186 \\
\hline
\end{tabular}

netic bead even in a fluidic environment such as water or blood where the buoyancy force is present.

A possible solution would be to provide a supplementary set of gradient coils designed to optimize the peak-topeak amplitude of the generated magnetic gradient instead of the current linearity of existing MRI gradient coils. Nevertheless, the present article presents a global proofof-concept of a software platform for MRI systems that includes propulsion, tracking, and control with user interfaces where the emphasis is placed on MRI-assisted realtime navigation. Such software architecture allowed us to investigate the limits of standard clinical MRI systems for this application. One of the major obstacles when considering custom made higher amplitude magnetic gradient coils and which must be considered when implementing such software architecture is the nerve stimulation threshold which can appear at higher amplitudes than the ones used for common imaging procedures. However, as noted in Refs. $(28,29)$, this threshold depends mainly on the rise time of the magnetic gradient, namely $\frac{d B}{d t}$. Empirical models and experimental data obtained from Ref. (29) stipulate that the mean $\frac{d B}{d t}$ threshold before experiencing peripheral nerve stimulation can be estimated as:

$$
\left(\frac{d B}{d t}\right)_{\text {threshold }}=54\left[1+\frac{132}{\tau}\right]
$$

where $\tau$ is the total ramp time of the magnetic gradient. In our case, a value of $\tau_{n a v}=1000 \mathrm{~ms}$ was used, leading to an estimate threshold of $61 \mathrm{~T} / \mathrm{s}$, which is approximately the required force for the levitation of the device. Since the force generated on the device is proportional to the magnetic gradient amplitude, a possible solution in order to limit such physiological phenomena could involve the alteration of the magnetic gradient rise so the $\frac{d B}{d t}$ threshold for peripheral nerve stimulation reaches a higher acceptable level.

\section{D Control on the MR Plate Phantom}

From Fig. 8 we observe a significant difference between the travel time on the $\mathrm{x}$ and $\mathrm{z}$ axis to reach a given waypoint. In fact, the $\mathrm{x}$ coordinate of the targeted waypoint is always reached before the $\mathrm{z}$ coordinate. Since all way- 
FIG. 9. Overview of the nine selected waypoints (red circles W1 through W9) and experimental positions (red and green dots) superimposed on the acquired angiogram. The successive directions of motion of the bead are illustrated by the yellow arrows. The navigation sequence is started with the bead at the W1 position and is moved along the phantom to reach the bottom branch (W5) then the upper branch (W7) and finally is directed to its starting position at W9. The red filled green dots represent the computed device positions along the phantom.

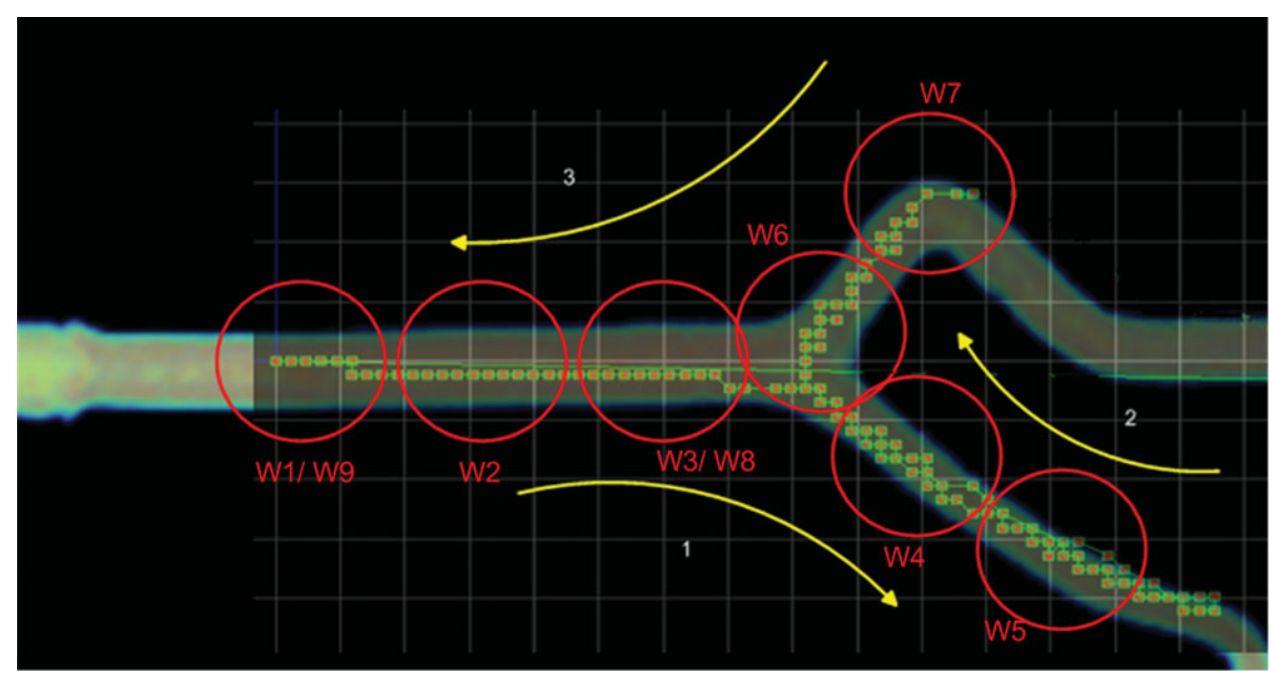

points (except for the last one) are located on a $45^{\circ}$ diagonal in the $\mathrm{x}-\mathrm{z}$ plane, we would expect a similar travel time to reach a given waypoint. We show from Ref. (13) that a ferromagnetic bead propelled on a surface inside an MRI in the presence of a DC magnetic field $B_{0}$ will slide in the direction of the $B_{0}$ orientation and will roll in the other directions (namely, on the $\mathrm{x}$ axis in our case), leading to two different friction coefficients between the steel and PMMA depending on the device displacement's orientation. Since the current 2D PID controller does not provide supplementary command correction, it would explain the difference in terms of travel time on the two different axes.

\section{D Control in MR Carotid Phantom with Quiescent Flow}

From Fig. 9 we see that some experimental position points seem to be located either on the edge or slightly outside the angiogram. This comes from the fact that when high propulsion gradients are applied the setup slightly vibrates because of the high amplitude variations in magnetic gra-

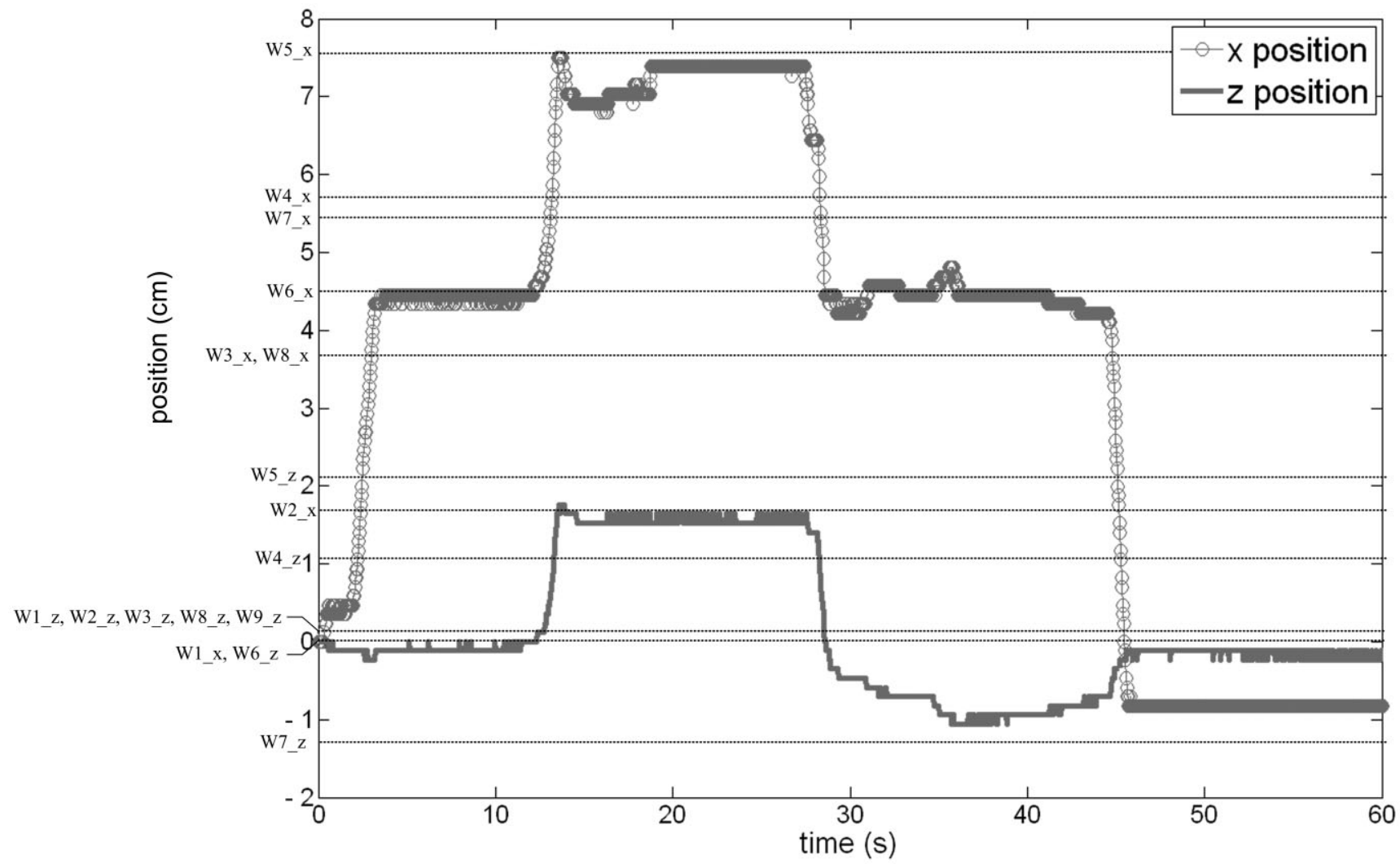

FIG. 10. Experimental data positions for the navigation along a set of nine waypoints in the Shelley MR phantom. Waypoint positions for the $\mathrm{x}$ and $\mathrm{z}$ axis are represented with a dashed line and are superimposed on the experimental positions. 
dients, leading to some small tracking imprecision. During the experiment the value of the quiescent flow was adjusted to prevent the bead from being stuck at certain waypoints (namely, waypoints 5 and 7). From Fig. 10 we see that the navigation of the bead is sometimes subject to short periods of stagnation on one or both axes. The explanation for such pause is the presence of a higher static friction coefficient between the ferromagnetic bead and the PMMA plate and is independent of the software architecture. This friction coefficient prevents the bead from moving in a given direction when magnetic gradients are applied during immobilization. After subsequent magnetic gradient peak, the bead starts oscillating and only the kinetic friction coefficient is considered allowing motion.

\section{Impact of Imaging Gradient}

If an imaging pulse sequence has to be considered during the navigation process, the impact of the imaging gradients on the device position has to be considered. The strongest magnetic gradient amplitudes are mostly found in ultrafast interventional or functional imaging pulse sequences where real-time imaging is a requirement. Magnetic gradients amplitudes as high as $25 \mathrm{mT} / \mathrm{m}$ with a duty cycle of $50 \%$ are to be considered. However, the short period during which the magnetic gradient is 'on' is on the order of hundreds of microseconds, leading to a clearly insufficient net average force application period for putting the bead into motion (not exceeding one-fourth of the gravitational attraction).

\section{CONCLUSION}

This study demonstrated that using special interconnected software modules, most clinical MRI systems can be adapted with no hardware modification as a dedicated interventional MRI system able to drive and control magnetic materials inside complex vasculature environments. A custom-made real-time pulse sequence used for the propulsion and tracking of the device is coupled with different software modules for the position analysis, command computation, and path planning operations necessary for the human free navigation of a $1.5 \mathrm{~mm}$ diameter chrome steel ferromagnetic sphere. Miniaturization of a such device to micro- or even nanomagnetic particles could lead to a new range of controllable targeted drug delivery or wireless biosensors for remote physiological data measurement and analysis.

\section{ACKNOWLEDGMENTS}

The authors thank S. Zuehlsdorff for support during the sequence development phase, E. Aboussouan and M. Mankiewicz for technical assistance, and G. Potvin for MRI assistance.

\section{REFERENCES}

1. Atalar E. MR-guided vascular interventions. IEEE Engineering in Medicine and Biology Society (EMBS). 2001; Oct 25-28. p 4056.
2. Green JD, Omary RA, Schirf BE, Tang R, Lu B, Gehl JA, Huang JJ, Carr JC, Pereles FS, Li D. Comparison of X-ray fluoroscopy and interventional magnetic resonance imaging for the assessment of coronary artery stenoses in swine. Magn Reson Med 2005;54:1094-1099.

3. Jerzewski A, Pattynama PMT, Steendijk P, Doornbos J, Roos A, Baan J. Development of an MRI-compatible catheter for pacing the heart: Initial in vitro and in vivo results. J Magn Reson Imaging 1996;6:948-949.

4. Bulte JWM. Magnetic nanoparticles as markers for cellular MR imaging. J Magnetism Magn Mater 2005;289:423-427.

5. McDannold N, Vykhodtseva N, Raymond S, Jolesz FA, Hynynen K. MRI-guided targeted blood-brain barrier disruption with focused ultrasound: Histological findings in rabbits. Ultrasound Med Biol 2005; 31:1527-1537.

6. Mulder WJM, Strijkers GJ, van Tilborg GAF, Griffioen AW, Nicolay K. Lipid-based nanoparticles for contrast-enhanced MRI and molecular imaging. NMR Biomed 2006;19:142-164.

7. Schmieder AH, Winter PM, Caruthers SD, Harris TD, Williams TA, Allen JS, Lacy EK, Zhang H, Scott MJ, Hu G, Robertson JD, Wickline SA, Lanza GM. Molecular MR imaging of melanoma angiogenesis with alpha_nu beta_3-targeted paramagnetic nanoparticles. Magn Reson Med 2005;53:621-627.

8. Martel S, Mathieu J-B, Felfoul O, Chanu A, Aboussouan E, Tamaz S, Pouponneau P, Beaudoin G, Soulez G, Yahia LH, Mankiewicz M. Automatic navigation of an untethered device in the artery of a living animal using a conventional clinical magnetic resonance imaging system. Appl Phys Lett 2007;90:114105.

9. Grady MS, Howard MA, Molloy JA, Ritter RC, Quate EG, Gillies GT, Nonlinear magnetic stereotaxis: three-dimensional, in vivo remote magnetic manipulation of a small object in canine brain. Med Phys 1990;17:405-415.

10. Grady MS, Howard MA, Ritter RC, Quate EG, Molloy JA, Lawson MA, Wika KG, Ramos PA, Gillies GT. Magnetic stereotaxis system for neurosurgical procedures. In: Proc 37th International Instrumentation Symposium, May 5-9 1991: Instrumentation in the Aerospace Industry, Proceedings of the ISA Aerospace Instrumentation Symposium 1991; 37:665-675.

11. Martel S, Mathieu JB, Felfoul O, Macicior H, Beaudoin G, Soulez G, Yahia LH. Adapting MRI systems to propel and guide microdevices in the human blood circulatory system. IEEE Engineering in Medicine and Biology Society (EMBS). 2004; San Francisco. p 1044-1047

12. Mathieu JB, Martel S, Yahia LH, Soulez G, Beaudoin G. MRI systems as a mean of propulsion for a microdevice in blood vessels. IEEE 2003. p 3419-3422.

13. Mathieu J-B, Martel S, Yahia LH, Soulez G, Beaudoin G. Preliminary investigation of the feasibility of magnetic propulsion for future microdevices in blood vessels. Biomed Mater Eng 2005;15:367-374.

14. Mathieu JB, Beaudoin G, Martel S. Method of propulsion of a ferromagnetic core in the cardiovascular system through magnetic gradients generated by an MRI system. IEEE Trans Biomed Eng 2006;53: 292-299.

15. Elgort DR, Hillenbrand CM, Shaoxiaong Z, Wong EY, Rafie S, Lewin JS, Duerk JL. Image-guided and -monitored renal artery stenting using only MRI. J Magn Reson Imaging 2006;23:619-627.

16. Hillenbrand CM, Elgort DR, Wong EY, Reykowski A, Wacker FK, Lewin JS, Duerk JL. Active device tracking and high-resolution intravascular MRI using a novel catheter-based, opposed-solenoid phased array coil. Magn Reson Med 2004;51:668-675.

17. Graf H, Lauer UA, Berger A, Schick F. RF artifacts caused by metallic implants or instruments which get more prominent at $3 \mathrm{~T}$ : an in vitro study. Magn Reson Imaging 2005;23:493-499.

18. Graf H, Steidle G, Martirosian P, Lauer UA, Schick F. Effects on MRI due to altered rf polarization near conductive implants or instruments. Med Phys 2006;33:124-127.

19. Pauchard Y, Smith MR, Mintchev MP. Improving geometric accuracy in the presence of susceptibility difference artifacts produced by metallic implants in magnetic resonance imaging. IEEE Trans Med Imaging 2005;24:1387-1399.

20. Cho ZH, Kim DJ, Kim YK. Total inhomogeneity correction including chemical shifts and susceptibility by view angle tilting. Med Phys 1988;15:7-11.

21. Felfoul O, Mathieu JB, Beaudoin G, Martel S. In vivo MR-tracking based on magnetic signature selective excitation. Trans Med Imaging 2008; $27: 28-35$. 
22. Aboussouan E, Felfoul O, Mathieu JB, Beaudoin G, Martel S. Real-time projection based technique for tracking ferromagnetic devices. International Society for Magnetic Resonance Medicine. 2006; Seattle. p 637.

23. Aboussouan E, Martel S. High-precision absolute positioning of medical instruments in MRI systems. IEEE Engineering in Medicine and Biology Society (EMBS). New York; 2006. p 743-746.

24. Seppenwoolde JH, Viergever MA, Bakker CJG. Passive tracking exploit ing local signal conservation: the white marker phenomenon. Magn Reson Med 2003;50:784-790.

25. Tamaz S, Martel S. Impact of the MRI-based navigation system constraints on the step response using a PID controller. IEEE Engineering in Medicine and Biology Society (EMBS). 2005; p 5073-5076.
26. Mathieu JB, Beaudoin G, Martel S. Method of propulsion of a ferromagnetic core in the cardiovascular system through magnetic gradients generated by an MRI system. IEEE Trans Biomed Eng 2006;53: 292-299.

27. Tamaz S, Gourdeau R, Martel S. Bidimensional MRI-based navigation system using a PID controller. IEEE Engineering in Medicine and Biology Society (EMBS). New York 2006; p 4424-4427.

28. Ham CLG, Engels JML, Van de Wiel GT, Machielsen A. Peripheral nerve stimulation during MRI: effects of high gradient amplitudes and switching rates. J Magn Reson Imaging 1997;7:933-937.

29. King FK, Schaefer J. Spiral scan peripheral nerve stimulation. J Magn Reson Imaging 2000;12:164-170. 